\title{
Intensity-Modulated Optical Fiber Sensor for AC Magnetic Field Detection
}

\author{
Ivo M. Nascimento, J. M. Baptista, P. A. S. Jorge, Jose L. Cruz, and Miguel V. Andrés, Member, IEEE
}

\begin{abstract}
An erbium-doped $\left(\mathrm{Er}^{3+}\right)$ fiber optic laser is proposed for sensing alternated magnetic fields by measuring the laser intensity modulation. The sensor is fabricated using two partially overlapped narrow-band fiber Bragg gratings (FBGs) and a section of doped fiber in a Fabry-Perot configuration. Laser power stability and bandwidth are studied while changing the overlap. A bulk rod of TbDyFe, a magnetostrictive material, is glued to both the FBGs and the laser wavelength and power are modulated according to the magnetic field. Acquisition and processing are done using virtual instrumentation. Results have shown the possibility of detecting $11.18 \mu \mathrm{T}_{\mathbf{r m s}} / \sqrt{ } \mathrm{Hz}$ for an alternating magnetic field of $4.17 \mathrm{mT}_{\text {rms }}$.
\end{abstract}

Index Terms - Magnetic field sensor, magnetostrictive material, erbium doped fiber laser, intensity modulation.

\section{INTRODUCTION}

$\mathbf{F}$ IBER optic magnetic field sensors have been investigated over the years for current measurement. These sensors can be employed in power networks, replacing traditional transformers where catastrophic explosive failures can happen, offering high voltage immunity, light weight, large bandwidth and small size [1], [2]. Typically, optical magnetic field sensors are based on magnetic fluids, Lorentz force, Faraday and magnetostrictive effect.

For instance, a magnetic fluid comprises a liquid solution whose refractive index depends on the applied magnetic field.

Manuscript received May 11, 2015; revised August 13, 2015; accepted August 16, 2015. Date of publication August 20, 2015; date of current version October 2, 2015. This work was supported in part by the SMARTGRIDS Project under Grant NORTE-07-0124-FEDER-000056 through the North Portugal Regional Operational Program ON.2-O Novo Norte, under the National Strategic Reference Framework, within the European Regional Development Fund (FEDER), and in part by the National Funds within the Portuguese Funding Agency through Fundação para a Ciência e a Tecnologia (FCT). The work of I. M. Nascimento was supported by FCT under Grant SFRH/BD/80056/2011. The work of J. L. Cruz and M. V. Andrés was supported in part by the Ministerio de Economia y Competitividad of Spain, in part by Fondo FEDER, and in part by Generalitat Valenciana under Project TEC2013-46643-C2-1-R and Project PROMETEOII/2014/072.

I. M. Nascimento is with the Instituto de Engenharia de Sistemas e Computadores, Tecnologia e Ciência, Porto 4200, Portugal, and also with the Faculdade de Ciências, Universidade do Porto, Porto 4099-002, Portugal (e-mail: ivomac88@gmail.com).

J. M. Baptista is with the Instituto de Engenharia de Sistemas e Computadores, Tecnologia e Ciência, Porto 4200, Portugal, and also with the Centro de Competências de Ciências Exatas e de Engenharia, Universidade da Madeira, Funchal 9000-082, Portugal (e-mail: jmb@uma.pt).

P. A. S. Jorge is with the Instituto de Engenharia de Sistemas e Computadores, Tecnologia e Ciência, Porto 4200, Portugal (e-mail: pedro.jorge@fc.up.pt).

J. L. Cruz and M. V. Andrés are with the Department of Applied Physics and Electromagnetism, University of Valencia, Valencia 46010, Spain (e-mail: jose.1.cruz@uv.es; miguel.andres@uv.es).

Color versions of one or more of the figures in this letter are available online at http://ieeexplore.ieee.org.

Digital Object Identifier 10.1109/LPT.2015.2470135
By using a refractive index sensor, such as an LPG (Long Period Grating), the magnetic field can be quantified with a minimum detectable strength of $1 \mathrm{mT}$ [3]. In high power networks, the most common used sensing mechanism is the Faraday effect. Light propagating through a medium can be influenced by the application of an external magnetic field, parallel to light propagation, inducing a non-reciprocal rotation of the plane of vibration of linear polarized light. Sensors based on Faraday effect essentially employ fibers with multiple turns around the conductor or a bulk prism with high Verdet constant to improve the sensitivity [5].

On the other hand, optical fiber lasers combined with magnetostrictive materials or with an extra current carrying conductor (Lorentz force effect) can be used for magnetic field sensing. These sensors are attractive due to the narrow linewidth, low relative noise, high power and the possibility of emitting a single-longitudinal mode and a defined polarization state [6]. Also, the use of erbium doped fiber lasers are attractive since they enable a broad range of emission wavelengths in $\mathrm{C}$ and $\mathrm{L}$ communication bands [7]. A single mode fiber laser with a Michelson interferometric readout system was developed for magnetic field sensing, capable of detecting $1.5 \mu \mathrm{T} / \sqrt{ } \mathrm{Hz}$ [8]. The laser comprised a $\pi$-shift FBG (Fiber Bragg Grating) which experienced strain when a magnetic field was present. This configuration also required an extra current carrying conductor attached and parallel to the laser cavity. A similar approach was also proposed in [2] employing a laser with two polarizations and one longitudinal mode. The beat frequency between polarizations was linearly dependent on the applied magnetic field with a relation of $984.4 \mathrm{kHz} / \mathrm{pT}$ and the beat frequency without magnetic field was $636 \mathrm{MHz}$, requiring high bandwidth photodetectors.

Another approach employed was a cavity with an erbium doped fiber, a mirror and a FRM (Faraday Rotator Mirror) to ensure temperature and strain stability. The magnetic field had to be applied near the output mirror where a non-reciprocal circular birefringence was induced and a linear analyser, following the laser output, was used to interfere both circular polarizations. The polarization mode beat was proportional to the magnetic field and it was read using a fast photodetector and a electric spectrum analyzer (ESA) [9].

Moreover, magnetostrictive effect has also been exhibited with a distributed optical fiber laser and a magnetostrictive nickel wire and a passive interferometric readout system with a $3 \times 3$ coupler. A resolution of $0.03 \mathrm{mT}$ with a spatial resolution of $1 \mathrm{~m}$, defined by the interferometer, was attained [10]. Other configurations employing magnetostrictive materials were also studied for magnetic field sensing. A quasi-balanced passive 


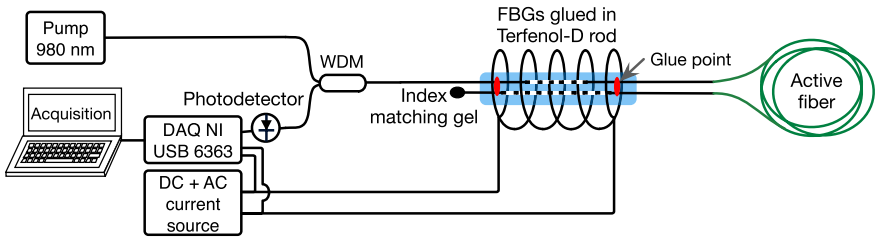

Fig. 1. Experimental setup of the erbium doped fiber laser and respective acquisition system.

interferometer was reported [11] where a Terfenol-D rod stretched a loop of $30 \mathrm{~m}$ in length with 115 fiber turns. A sensitivity of $69.83 \mathrm{mrad} / \mu \mathrm{T}$ and a resolution of $2.14 \mathrm{nT}_{\mathrm{RMS}} / \sqrt{ } \mathrm{Hz}$ were obtained at $200 \mathrm{~Hz}$.

In this document a simpler approach is reported which combines the intrinsic advantages of the optical fiber lasers, namely, high power and narrow spectral band. A magnetostrictive rod of TbDyFe (also known as Terfenol-D) is glued to the laser cavity mirrors (FBGs), modulating both FBGs as function of the applied magnetic field, hence the output power of the laser. Besides an easy fabrication, the sensor has a simple interrogation technique based on the laser intensity modulation introduced by the applied magnetic field, without requiring the use of more complex systems such as readout interferometers [8], [10], [11]. The output signal is processed to provide the quotient between the time dependent component and the continuous wave component, so that the readout is independent of the absolute laser power or the transmission loss. The magnetostrictive transducer is biased by a constant filed to achieve optimal response. Furthermore, the fabrication of the key element of sensing setup is straightforward requiring only two spectrally identical FBGs glued side-by side in the magnetostrictive material.

\section{PRINCIPLE}

Fig. 1 represents the experimental setup, the laser is a Fabry-Perot cavity having an erbium doped fiber as gain medium and two fiber gratings as mirrors. The mirrors are held by a magnetostricive transducer, which is subjected to a bias DC magnetic field and to the AC field to be measured. The small variation the field introduces in the gratings spectra produces a variation of the output power, which is detected and processed to recover the amplitude of the time dependent field. Simultaneously, the time variant electric current signal, proportional to the applied magnetic field, is also recorded.

\section{RESULTS}

\section{A. Laser Development and Characterization}

Two narrowband gratings were fabricated for this application in a Boron codoped photosensitive fiber, one had $3.6 \mathrm{~cm}$ in length and the other $6.7 \mathrm{~cm}$. The former had a bandwidth $37.24 \mathrm{~nm}$ and a reflectivity of $88 \%$ at $1530.939 \mathrm{~nm}$, the latter had a bandwidth of $27.58 \mathrm{pm}$ and reflectivity of $98 \%$ at $1531.066 \mathrm{~nm}$. The wavelengths were chosen near the emission peak of Erbium where a small variation of their spectral characteristics will result in a large

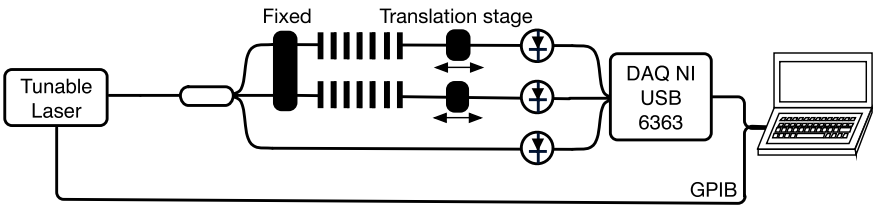

Fig. 2. Experimental setup for tuning the FBGs.

variation of the output power. Both FBGs had to be tuned in order to form the laser cavity and achieve laser emission. A bandwidth of $27.58 \mathrm{pm}$ and $37.24 \mathrm{pm}$ was measured for the FBG with higher and lower reflectivity, respectively. Moreover, to manufacture narrowband FBGs with high reflectivity, the modulation index contrast had to be low, but the length of the FBGs had to be increased.

Between both FBGs, a piece of Fibercore Erbium doped fiber is used as the gain medium. Two fibers of different Erbium concentrations were tested, the M-5 and the M-12 (whose absorptions are 6.97 and $17.28 \mathrm{~dB} / \mathrm{m}$ at $1531 \mathrm{~nm}$ respectively, corresponding to $\mathrm{Er}^{3+}$ concentrations of 300 and $1000 \mathrm{ppm}$ approximately). As it is explained later, best immunity to vibrations was achieved with the M-5 fiber. The final prototype had a piece of $6.8 \mathrm{~m}$ of M-5 fiber resulting in a cavity length of $9 \mathrm{~m}$. Each FBG was glued side by side in two points, distant $9 \mathrm{~cm}$ apart, in a Terfenol-D (composition $\left.\mathrm{Tb}_{0.27} \mathrm{Dy}_{0.73} \mathrm{Fe}_{2}\right)$ rod having a diameter of $0.5 \mathrm{~cm}$ and a length of $10 \mathrm{~cm}$. The laser output is seen up-stream, free of pump power; and in the down-stream end of the fiber, index-matching gel is used to avoid back reflection capable of introducing noise in the laser cavity. The magnetic field is generated with an inductor and the amplitude of the magnetic field is controlled using a sinusoidal current source with an adjustable DC offset.

The acquisition system is composed by a photodetector with $10 \mathrm{MHz}$ bandwidth and a 16 bits analogue-digital converter from NI (National Instruments) with $305 \mu \mathrm{V}$ resolution and 2 Mbps bandwidth, which reads the laser output power which is in turn dependent on the magnetic field. The signals read are filtered with a band-pass filter of $5 \mathrm{~Hz}$ bandwidth with a LabVIEW software.

The gratings were partially overlapped with strain so that the laser had a narrow emission line and enhanced intensity dependence on the spectral shape (hence more sensitivity to the magnetic field). To overlap the gratings Bragg wavelength, the setup in Fig. 2 was used. A tunable laser controlled with a computer, via GPIB (General Purpose Interface Bus) is used to simultaneously scan both FBGs spectrum in transmission with $1 \mathrm{pm}$ resolution. A third arm of the $3 \times 3$ coupler is used as a reference, to eliminate dependence on any laser output power variation.

A translation stage for each FBG is used to tune both FBGs and consequently control the Bragg wavelength overlap. In Fig. 3 it is shown the spectrum of the detuned FBGs with an overlap of $17.32 \mathrm{pm}$, at half power $(-3 \mathrm{~dB})$.

When the intended overlap is achieved the laser configuration shown in Fig. 1 is assembled. A section of fibercore M-12 active fiber of $0.5 \mathrm{~m}$ was used as the gain medium, 


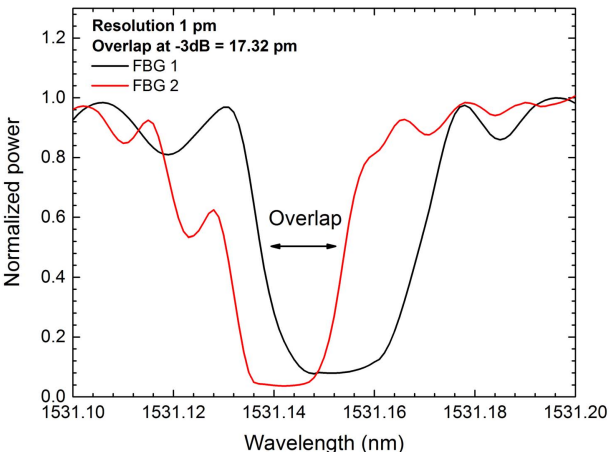

Fig. 3. FBGs with a 17.32 pm overlap.

TABLE I

POWER AND LASER LINEWIDTH AS FUNCTION OF THE FBG OVERLAP

\begin{tabular}{lll}
\hline \hline Overlap $(\mathrm{pm})$ & \multicolumn{1}{c}{ Power $(\mathrm{mW})$} & Laser linewidth $(\mathrm{MHz} / \mathrm{pm})$ \\
\hline 17.32 & 14.25 & $227 / 1.78$ \\
10.73 & 18 & $207 / 1.62$ \\
1 & 20 & $152 / 1.18$ \\
\hline \hline
\end{tabular}

resulting in a cavity length of $1.5 \mathrm{~m}$. In Table I it is shown the laser linewidth and optical power as function of the overlapped FBGs. When the overlap raises the laser linewidth also increases and the peak optical power decreases because the reflectivity at the center of the gratings spectrum is higher. The linewidth was measured using an ESA, a $50 \mathrm{GHz}$ photodetector bandwidth and a laser tuned closed to the optical fiber laser wavelength emission.

Power stability was also analyzed using a photodetector and an oscilloscope. For overlaps smaller than $1 \mathrm{pm}$, we observed that any acoustic vibration coupled into the laser cavity gave rise to an unstable emission regime. Moreover the ESA spectrum revealed only three longitudinal modes for this configuration. To minimize this effect a section of $2 \mathrm{~m}$ of SMF was introduced in the laser cavity (resulting in a cavity length of $3.5 \mathrm{~m}$ ) with the goal of increasing the number of longitudinal modes. The results showed a more stable laser, with no pulsing but the power fluctuated (slow drift) up to $10 \%$, with acoustic vibrations.

The ultimate laser configuration is the same as the one shown in Fig. 1 but the $0.5 \mathrm{~m}$ of M-12 fiber was replaced by a section of $6.8 \mathrm{~m}$ of M-5 fiber. The laser has now less gain and more length (hence more longitudinal modes) resulting in better immunity to acoustic vibrations. In this configuration no drift or instability was observed in the presence of vibrations. Also, a detuning of $13.14 \mathrm{pm}$ was adjusted for both FBGs by controlling the translation stages and then glued to the Terfenol-D rod, side by side and with glue points displaced $9 \mathrm{~cm}$ apart. A laser linewidth of $254 \mathrm{MHz}, 1.99 \mathrm{pm}$ at $1531.4 \mathrm{~nm}$ was obtained, with two orthogonal polarizations and several longitudinal modes, as shown in Fig. 4 (left).

The laser has a threshold of $21 \mathrm{~mW}$ and a maximum output power of $110 \mathrm{~mW}$ for $546 \mathrm{~mW}$ pump as shown in Fig. 4 (right). Furthermore, vibrations introduced to the laser cavity showed it was power stable.
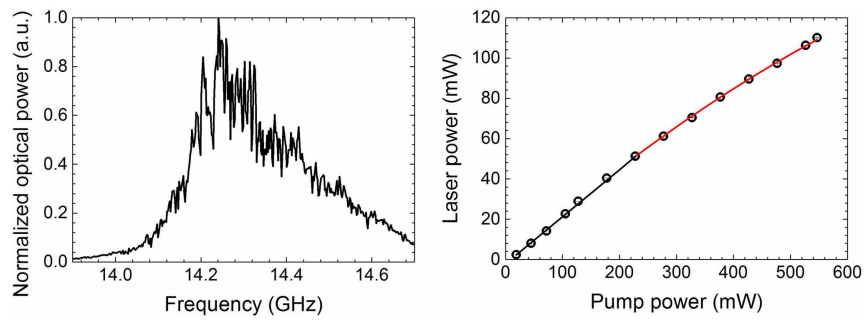

Fig. 4. Laser spectrum measured with an electrical spectrum analyzer (left) and output power as function of the pump power (right).

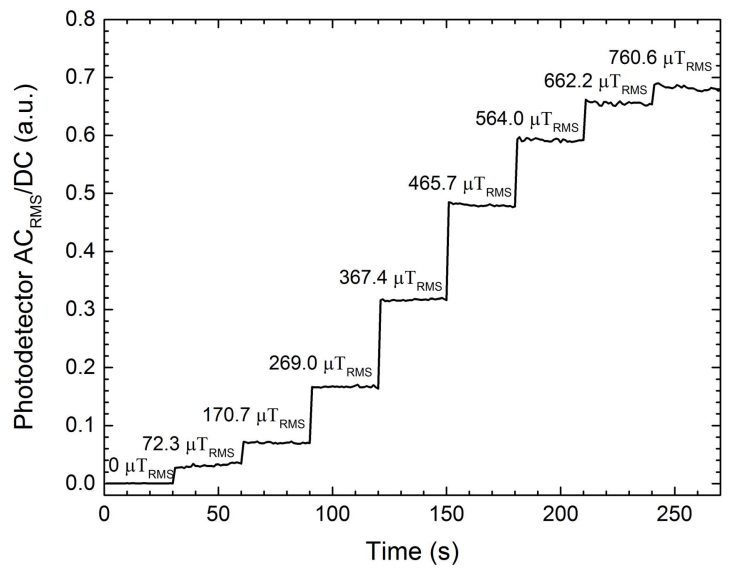

Fig. 5. AC power change normalized to the average emitted power as function of time for different applied AC magnetic fields. The bias DC magnetic field was $4.56 \mathrm{mT}$ for this test.

\section{B. Magnetic Field Measurement}

Following the laser characterization, the sensor response to magnetic field measurement was inspected using the setup presented in Fig. 1. Although the laser is power stable when an AC magnetic field is present, the reflectivity of the laser mirrors is modulated in wavelength and the laser output is also modulated in power, showing no pulsing. Having this effect into account, several constant and alternating (DC and $\mathrm{AC}$ ) magnetic fields were applied at $20 \mathrm{~Hz}$, and the power modulation was analyzed at 20 and $40 \mathrm{~Hz}$ with and without a magnetic bias field, respectively. In Fig. 5 it is shown the laser normalized $\mathrm{AC}$ response as function of the $\mathrm{AC}$ magnetic field, for a DC magnetic field of $4.56 \mathrm{mT}$. The laser emits an amplitude modulated wave. However, the quotient between the RMS value and the average value of the output signal is independent on the optical power, therefore independent on transmission loss or pump instabilities. Since processing makes the AC modulation power independent and the values shown in each step correspond to the value of the applied AC magnetic field.

Due to the non-linearity of the Terfenol-D response, it is expected the sensor response to $\mathrm{AC}$ magnetic fields will be dependent on the biasing constant magnetic field applied. Two independent measurements were done and are shown in Fig.6. The average and standard deviation of both measurements are also indicated, corresponding to the normalized $\mathrm{AC}$ response as function of the alternating magnetic field. Better sensitivities are attained for a constant magnetic field 


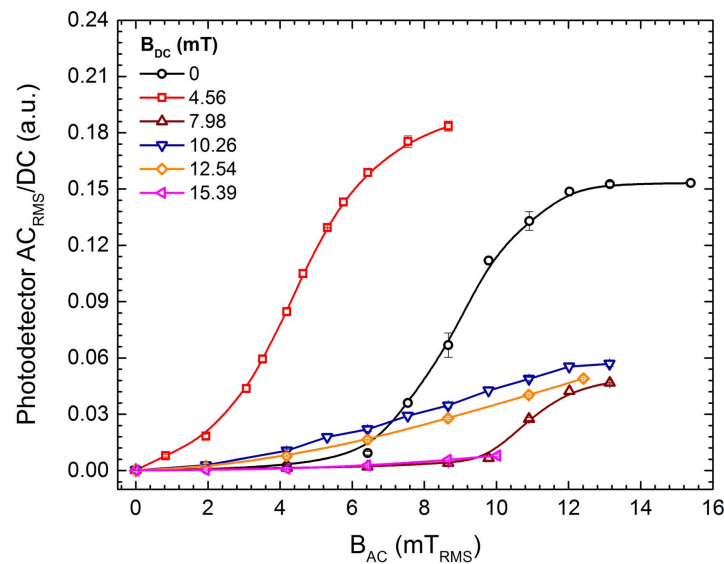

Fig. 6. Normalized AC power change as function of the applied AC magnetic field, for several bias magnetic fields.

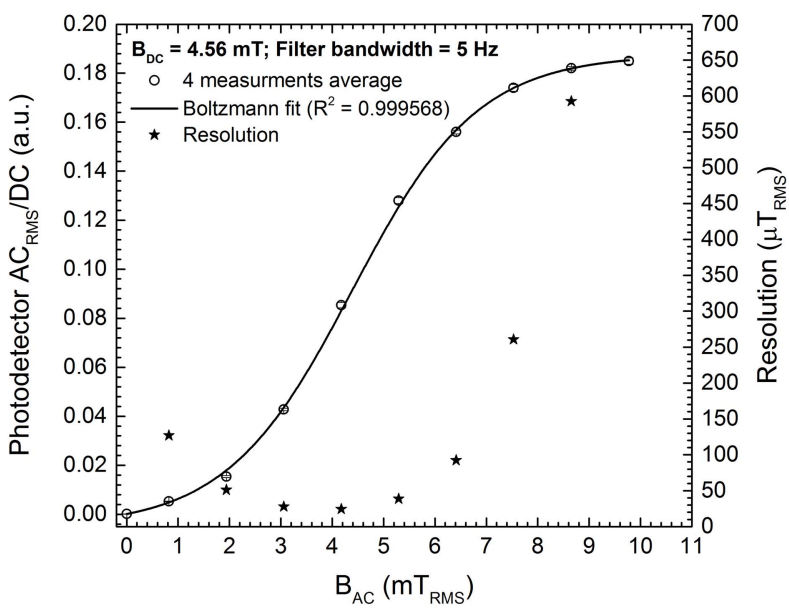

Fig. 7. Normalized AC power change as function of the applied AC magnetic field, for a DC magnetic field of $4.56 \mathrm{mT}$.

of 4.56 and $0 \mathrm{mT}$, enabling measuring up to 8.67 and $12.02 \mathrm{mT}_{\mathrm{RMS}}$, respectively. Moreover, for a constant field of $10.26 \mathrm{mT}$ a linear response is obtained ranging from 0 to $12.02 \mathrm{mT}_{\mathrm{RMS}}$, however the sensitivity is lower than the previous cases.

Considering the best result, obtained with a constant magnetic field bias of $4.56 \mathrm{mT}$, four independent measurements were repeated, from which the stability of the sensor was evaluated (Fig. 7). The worst resolutions obtained in each point of the calibration curve are then shown. The best resolutions obtained are 28 and $25 \mu \mathrm{T}_{\mathrm{RMS}}$ (12.52 and $\left.11.18 \mu \mathrm{T}_{\mathrm{RMS}} \sqrt{ } \mathrm{Hz}\right)$ for an alternating magnetic field of 3.05 and $4.17 \mathrm{mT}_{\mathrm{RMS}}$, respectively. This range also corresponds to the region with higher sensitivity, observed in Fig. 6. Outside this range, distortion increases and the worst resolution was $592.7 \mu \mathrm{T}_{\mathrm{RMS}}$ for an applied AC magnetic field of $8.65 \mathrm{mT}_{\mathrm{RMS}}$.

Comparing sensors stated in the state of the art with similar materials and working principle [8], [11] better resolutions are attained but it comes from the use of a more complex solution. However, the resolution achieved with the developed configuration is good and it can be improved in a future work combining the information of power and wavelength modulation.

\section{CONCLUSION}

In this letter a narrow band optical fiber laser for magnetic field measurement is proposed. This configuration provides the possibility of measuring alternating magnetic fields by merely measuring the normalized $\mathrm{AC}$ power modulation and does not require an interferometer or other kind of wavelength meter.

The two Bragg gratings mirrors of the laser cavity were modulated in wavelength by the Terfenol-D rod, as a result of the applied AC magnetic field, the emission power is also modulated. Taking advantage of this effect, the laser normalized $\mathrm{AC}$ power response was analysed using different constant and alternating magnetic fields. Owing to intrinsic response of the magnetostrictive material results, the sensor showed different sensitivities according to the operation point, defined by the bias magnetic field. Better sensitivities were achieved with a constant magnetic field of $4.56 \mathrm{mT}$ and worst results were attained for 10.26 and $12.54 \mathrm{mT}$. Considering the best result achieved, four calibration curves were made, demonstrating low errors between independent measurements. The best resolution was $25 \mu \mathrm{T}_{\mathrm{RMS}}\left(11.18 \mu \mathrm{T}_{\mathrm{RMS}} / \sqrt{ } \mathrm{Hz}\right)$ in the vicinity of an alternating magnetic field of $4.17 \mathrm{mT}_{\mathrm{RMS}}$.

\section{REFERENCES}

[1] T. W. MacDougall, D. R. Lutz, and R. A. Wandmacher, "Development of a fiber optic current sensor for power systems," in Proc. IEEE Power Eng. Soc. Transmiss. Distrib. Conf., Sep. 1991, pp. 336-341.

[2] B.-O. Guan and S.-N. Wang, "Fiber grating laser current sensor based on magnetic force," IEEE Photon. Technol. Lett., vol. 22, no. 4, pp. 230-232, Feb. 15, 2010.

[3] X. Li and H. Ding, "All-fiber magnetic-field sensor based on microfiber knot resonator and magnetic fluid," Opt. Lett., vol. 37, no. 24, pp. 5187-5189, Dec. 2012.

[4] F. Rahmatian and J. N. Blake, "Applications of high-voltage fiber optic current sensors," in Proc. Power Eng. Soc. General Meeting, Jun. 2006, pp. $1-6$.

[5] X. Deng, Z. Li, Q. Peng, J. Liu, and J. Tian, "Research on the magneto-optic current sensor for high-current pulses," Rev. Sci. Instrum., vol. 79, no. 8, p. 083106, Aug. 2008.

[6] Y. Zhao et al., "Research on a novel composite structure $\mathrm{Er}^{3+}$-doped DBR fiber laser with a П-phase shifted FBG," Opt. Exp., vol. 21, no. 19, pp. 22515-22522, 2013.

[7] Y. O. Barmenkov, A. V. Kir'yanov, P. Pérez-Millán, J. L. Cruz, and M. V. Andrés, "Experimental study of a symmetrically-pumped distributed feed-back erbium-doped fiber laser with a tunable phase shift," Laser Phys. Lett., vol. 5, no. 5, pp. 357-360, 2008.

[8] G. A. Cranch, G. M. Flockhart, and C. K. Kirkendall, "DFB fiber laser magnetic field sensor based on the Lorentz force," in Proc. Opt. Fiber Sensors, 2006, pp. 1-4, Paper TuC2.

[9] J. S. Park, S. H. Yun, S. J. Ahn, and B. Y. Kim, "Polarizationand frequency-stable fiber laser for magnetic-field sensing," Opt. Lett., vol. 21, no. 14, pp. 1029-1031, Jul. 1996.

[10] A. Masoudi and T. P. Newson, "Distributed optical fiber dynamic magnetic field sensor based on magnetostriction," Appl. Opt., vol. 53, no. 13, pp. 2833-2838, 2014.

[11] F. Chen, Y. Jiang, and L. Jiang, " $3 \times 3$ coupler based interferometric magnetic field sensor using a TbDyFe rod," Appl. Opt., vol. 54, no. 8, pp. 2085-2090, 2015. 